\title{
A Rudin-Osher-Fatemi Model-Based Pansharpening Approach Using RKHS and AHF Representation
}

\author{
Jun Liu ${ }^{1}$, Liang-Jian Deng ${ }^{2, *}$, Faming Fang ${ }^{3}$ and Tieyong Zeng ${ }^{4}$ \\ ${ }^{1}$ School of Mathematics and Statistics, Northeast Normal University, Changchun, \\ Jilin 130024, P.R. China. \\ ${ }^{2}$ School of Mathematical Sciences, University of Electronic Science and Technology \\ of China, Chengdu, Sichuan 611731, P.R. China. \\ ${ }^{3}$ Department of Computer Science, East China Normal University, Shanghai \\ 201101, P.R. China. \\ ${ }^{4}$ Department of Mathematics, Chinese University of Hong Kong, Hong Kong.
}

Received 15 August 2018; Accepted (in revised version) 6 November 2018.

\begin{abstract}
A pansharpening method based on the Rudin-Osher-Fatemi model and using a reproducing kernel Hilbert space along with the approximated Heaviside function is developed. The corresponding minimisation problem is solved by the alternating direction method of multipliers. Numerous numerical experiments with Pléiades and IKONOS satellite datasets demonstrate the efficiency of the method in preserving spectral and spatial information and show its superiority to other approaches.
\end{abstract}

AMS subject classifications: 47N10, 65C20, 68U10

Key words: Multi-spectral image, reproducing kernel Hilbert space, pansharpening, approximated Heaviside function.

\section{Introduction}

The remote sensing images acquired by earth resource satellites - e.g. by QuickBird, IKONOS, Landsat, are used to monitor the surface of the earth. They play an important role in military intelligence, environmental monitoring, land-cover classification and so on. Such images usually consist of two components - viz. the multi-spectral (MS) and panchromatic (PAN) images which, respectively, provide spectral and spatial information. At the same time, the high-resolution MS images are used in various applications, including pattern classification, land-cover management, environmental monitoring, weather forecasting, and topographic map updating [7]. Nevertheless, physical and technical limitations often lead to low resolution MS images. Therefore, the development of a super-resolution technique is needed. The super-resolution of an MS image consists in deriving an HRMS

\footnotetext{
${ }^{*}$ Corresponding author. Email addresses: liuj292@nenu.edu.cn (J. Liu), liangjian.deng@ uestc.edu.cn (L. Deng) 
image in both spatial and spectral domains by blending the geometric details of PAN image and the rich spectral information of MS image. This fusion process is referred to as pansharpening.

The pansharpening has attracted intensive attention in remote sensing. According to Amro et al. [6], the pansharpening methods can be roughly classified into five categories viz. Component Substitution (CS), Relative Spectral Contribution (RSC), High-Frequency Injection (HFI), Image Statistics Based (ISB), and Multiresolution Analysis (MRA). The CS methods carry out spectral transformation, do the upsampling of a low resolution multispectral (LRMS) images and substitute the corresponding components by high-resolution PAN images. Classical CS methods include the intensity-hue-saturation $[11,40]$, the principal component analysis [34] and the Gram-Schmidt (GS) spectral sharpening [30]. On the other hand, the RSC methods can be considered as an alternative to CS pansharpening methods, which works with linear combinations of spectral bands instead of substitutions. The HFI methods proposed by Schowengerdt [37], add the high-frequency content of the PAN image to upsampled LRMS images. The ISB methods use statistical information of LRMS and PAN images during the pansharpening — cf. [24]. The MRA approach decomposes low resolution multi-spectral and PAN images into different spatial levels and improves the spatial details of low resolution multi-spectral images by adding high frequencies from the PAN image. The corresponding approaches include Laplacian pyramid [10], à-trous wavelet transform [38] and additive wavelet luminance proportional method [33].

In addition to the already mentioned approaches, another possibility to improve MS images is provided by variational methods. The main idea of these methods consists in the construction of an energy functional, which utilises prior information and assumptions, and in finding the optimal solutions of the corresponding optimisation problem. In particular, Ballester et al. [8] introduced a variational pansharpening model named $P+X S$-method. It is based on the assumption that the geometry of the MS components are contained in the topographic map of the PAN image, which in turn is the linear combination of HRMS components. However, such interaction between PAN and MS images at the high spatial resolution produces spectral distortion [39]. Möller et al. [32] proposed a variational scheme with integrate wavelet fusion into the $P+X S$-method. Restaino et al. [35] applied mathematical morphology to pansharpening and demonstrated the efficiency of this approach for several data sets. Papers $[7,21]$ provide an extensive review of pansharpening methods. Jiang et al. [28] used a hyper-Laplacian based pansharpening model. It estimates the final pan-sharpened image directly, but not the coefficients of a basis as we do here. In addition, differential operators are applied to the difference of latent combined HRMS and panchromatic images, and a hyper-Laplacian regularisation is used. On the other hand, in order to simultaneously fuse panchromatic and multispectral images, Zhang et al. [47] presented a framelet based iterative pansharpening method. Unlike [28,47], this model is based on a reproducing kernel Hilbert space and the Heaviside function, where the former keeps the main image data and the later deals with the image edges. The coefficients in the reproducing kernel Hilbert space and the Heaviside basis are used to foresee unknown pixels, thus increasing the image resolution. In addition, an iterative strategy to enhance the performance of image pansharpening is employed. 
The previous considerations show that optimisation models play an important role in construction of high-quality pansharpening methods. Here we propose an effective pansharpening model based on the following assumptions:

1. The gradient of the high-resolution PAN image is a linear combination of the gradients of different bands of the desired HRMS image. This assumption helps to preserve spatial information in HRMS images.

2. Each band of HRMS and PAN images can be represented by a redundant basis from a reproducible kernel Hilbert space and the set of approximated Heaviside functions.

The outline of this paper is as follows. In Section 2, we briefly introduce a reproducible kernel Hilbert space and approximated Heaviside functions. A new model and the corresponding algorithm are presented in Section 3. Numerical experiments are carried out in Section 4 and Section 5 contains conclusion remarks.

\section{Reproducible Kernel Hilbert Space and Approximated Heaviside Functions}

Deng et al. [18] represented the smooth part of an image by the elements of a reproducing kernel Hilbert space (RKHS) and the edges by the set of approximated Heaviside functions (AHF). Let $f$ be a function defined on a continuous domain. The high and lowresolution images can be considered as the fine and coarse discretisations of $f$, respectively. The function $f$ can be usually represented as a sum $f=f_{s}+f_{e}$ of smooth $f_{s}$ and edge $f_{e}$ components. As was shown in [18], every $f_{s}$ belongs to RKHS and $f_{e}$ describes the edges at various locations with different orientations represented by a group of redundant AHF.

Wahba [43] proved that an optimal spline smooth approximation of $f$ can be obtained by solving a variational minimisation problem. The smooth part $f_{s}$ can be determined by the Dunchon method [20] — viz.

$$
f_{s}(t)=\sum_{i=1}^{m} \xi_{i} \phi_{i}(t)+\sum_{j=1}^{n} \zeta_{j} E_{k}\left(t, t_{j}\right),
$$

where functions $\phi_{i}$ constitute a basis in a polynomial space, $m$ is the dimension of this space, $E_{k}\left(t, t_{j}\right)$ Green's function for the $k$-iterated Laplacian, $n$ the number of pixels in a low-resolution image, and $\xi_{i}, \zeta_{j}$ are corresponding coefficients.

The edge part $f_{e}$ of $f$ can be represented in the form

$$
f_{e}=\sum_{j=1}^{M} \sum_{i=1}^{n} \gamma_{j} \psi\left(\left(\cos \theta_{j}, \sin \theta_{j}\right) \cdot t+c_{i}\right),
$$

where $M$ denotes the number of orientations, $\theta_{j}$ and $c_{i}$ control the orientations of the edges at different locations and

$$
\psi(x)=\frac{1}{2}+\frac{1}{\pi} \arctan \left(\frac{x}{\tau}\right)
$$


is the approximation of the Heaviside function (AHF) [18].

Let $\mathbf{u} \in \mathbb{R}^{n}$ be the vector-form of a low resolution image and let $\mathbf{T}^{l} \in \mathbb{R}^{n \times m}, \mathbf{K}^{l} \in \mathbb{R}^{n \times n}$, and $\Psi^{l} \in \mathbb{R}^{n \times M n}$ be, respectively, matrix representations for the discretisation operators generated by the functions $\phi, E_{3}$ and $\psi-$ cf. [18]. Analogously, for the fine grid, the matrices related to HR image $\mathbf{U}$ are referred to as $\mathbf{T}^{h} \in \mathbb{R}^{\tilde{n} \times m}, \mathbf{K}^{h} \in \mathbb{R}^{\tilde{n} \times n}$, and $\Psi^{h} \in \mathbb{R}^{\tilde{n} \times M n}$, respectively. If a HR image has the form $\mathbf{U}=\mathbf{T}^{h} \mathbf{d}+\mathbf{K}^{h} \mathbf{c}+\Psi^{h} \boldsymbol{\beta}$, then the coefficients $\mathbf{d}$, $\mathbf{c}$, and $\boldsymbol{\beta}$ can be derived by solving the minimisation problem

$$
\min _{\mathbf{b}, \mathbf{c}, \boldsymbol{\beta}} \frac{1}{2 n}\left\|\mathbf{u}-\left(\mathbf{T}^{l} \mathbf{d}+\mathbf{K}^{l} \mathbf{c}+\Psi^{l} \boldsymbol{\beta}\right)\right\|_{2}^{2}+\lambda \mathbf{c}^{T} \mathbf{K}^{l} \mathbf{c}+\alpha\|\boldsymbol{\beta}\|_{1}
$$

In what follows, we apply RKHS and AHF for pansharpening with a specially designed variational minimisation problem.

\section{A New Model and Algorithm}

We first introduce necessary notations. Let $P \in \mathbb{R}^{m_{2} n_{2} \times 1}$ be the vector form of a highresolution PAN image, $\mathbf{u}=\left(\mathbf{u}_{1}, \cdots, \mathbf{u}_{N}\right), \mathbf{u}_{i} \in \mathbb{R}^{m_{1} n_{1} \times 1}, i=1,2, \cdots, N$ the low resolution multi-spectral image with the band number $N$, and $\mathbf{U}=\left(\mathbf{U}_{1}, \cdots, \mathbf{U}_{N}\right), \mathbf{U}_{i} \in \mathbb{R}^{m_{2} n_{2} \times 1}$, $i=1,2, \cdots, N$ the HRMS image. Since every band in the HRMS image is supposed to be represented by a basis in RKHS and AHF, we have to find the corresponding coefficients.

Let us recall that the variational pansharpening model presented in [23] is based on the assumption that the gradient of PAN image is a linear combination of the pan-sharpened image bands. In addition, the efficiency of the $\ell_{1}$-norm as fidelity term has been demonstrated in [14]. Our work uses the assumptions mentioned. In addition, we employ the basis introduced in Section 2. Thus let us consider the minimisation problem

$$
\begin{aligned}
\min _{\mathbf{b}, \mathbf{c}, \boldsymbol{\beta}} \frac{1}{2 N_{u m}} \sum_{i=1}^{N}\left\{\left\|\left(\mathbf{T}^{h} \mathbf{d}_{i}+\mathbf{K}^{h} \mathbf{c}_{i}+\Psi^{h} \boldsymbol{\beta}_{i}\right)-\tilde{\mathbf{U}}_{i}\right\|_{2}^{2}+\frac{\mu}{2} \mathbf{c}_{i}^{T} \mathbf{K}^{l} \mathbf{c}_{i}+\lambda_{1}\left\|\boldsymbol{\beta}_{i}\right\|_{1}\right\} \\
+\lambda_{2}\left\|\nabla\left(\sum_{i=1}^{N} \sigma_{i}\left(\mathbf{T}^{h} \mathbf{d}_{i}+\mathbf{K}^{h} \mathbf{c}_{i}+\Psi^{h} \boldsymbol{\beta}_{i}\right)\right)-\nabla P\right\|_{1},
\end{aligned}
$$

where $N_{u m}=m_{2} \cdot n_{2} \cdot N$ is the number of pixels in HRMS image, $\mu, \lambda_{1}$ and $\lambda_{2}$ are regularisation parameters, $\sigma_{i} \geq 0, i=1,2, \cdots, N$ the mixing coefficients, $\tilde{\mathbf{U}}=\left(\tilde{\mathbf{U}}_{1}, \tilde{\mathbf{U}}_{2}, \cdots, \tilde{\mathbf{U}}_{N}\right)$ the approximated HRMS image obtained by the GS method [30], and $\mathbf{K}^{l} \in \mathbb{R}^{m_{1} n_{1} \times m_{1} n_{1}}, \mathbf{T}^{h} \in$ $\mathbb{R}^{m_{2} n_{2} \times M}, \mathbf{K}^{h} \in \mathbb{R}^{m_{2} n_{2} \times m_{1} n_{1}}, \Psi^{h} \in \mathbb{R}^{m_{2} n_{2} \times m}$.

The minimisation problem (3.1) can be solved by various optimisation methods, including the alternating direction method of multipliers (ADMM) $[22,25,27,31]$ and a fashionable primal-dual approach [13]. In this paper, we apply the ADMM method, which is a splitting version of the augmented Lagrangian method. For this, we first rewrite (3.1) in a matrix-vector form

$$
\min _{\mathbf{x}} \frac{1}{2 N_{u m}}\left\{\|\mathbf{A} \mathbf{x}-\mathbf{y}\|_{2}^{2}+\frac{\mu}{2}\|\mathbf{B} \mathbf{x}\|_{2}^{2}+\lambda_{1}\|\mathbf{C} \mathbf{x}\|_{1}\right\}+\lambda_{2}\|\nabla(\mathbf{D x}-P)\|_{1}
$$


where

$$
\begin{aligned}
& \mathbf{y}=\left(\tilde{\mathbf{U}}_{1}^{T}, \tilde{\mathbf{U}}_{2}^{T}, \cdots, \tilde{\mathbf{U}}_{N}^{T}\right) \in \mathbb{R}^{m_{2} n_{2} N \times 1}, \\
& \mathbf{x}=\left(\mathbf{d}_{1}, \mathbf{c}_{1}, \boldsymbol{\beta}_{1}, \cdots, \mathbf{d}_{N}, \mathbf{c}_{N}, \boldsymbol{\beta}_{N}\right) \in \mathbb{R}^{\left(M n_{2}+m_{1} n_{1}+m\right) N \times 1}, \\
& \mathbf{A}:=\operatorname{diag}(G, G, \cdots, G) \in \mathbb{R}^{m_{2} n_{2} N \times\left(m+m_{1} n_{1}+M n_{2}\right) N}, \\
& G=\left(\mathbf{T}^{h}, \mathbf{K}^{h}, \Psi^{h}\right), \quad \mathbf{B}:=\operatorname{diag}(F, F, \cdots, F), \quad F=\left(O_{1},\left(\mathbf{K}^{l}\right)^{1 / 2}, O_{2}\right), \\
& \mathbf{C}:=\left(O_{3}, O_{4}, I\right), \quad \mathbf{D}:=\left(\sigma_{1} \mathbf{T}^{h}, \sigma_{1} \mathbf{K}^{h}, \sigma_{1} \Psi^{h}, \cdots, \sigma_{N} \mathbf{T}^{h}, \sigma_{N} \mathbf{K}^{h}, \sigma_{N} \Psi^{h}\right),
\end{aligned}
$$

$I \in \mathbb{R}^{m \times m}$ is the unit matrix and $O_{1} \in \mathbb{R}^{m_{1} n_{1} \times m}, O_{2} \in \mathbb{R}^{m_{1} n_{1} \times M n_{2}}, O_{3} \in \mathbb{R}^{M n_{2} \times M n_{2}}, O_{4} \in$ $\mathbb{R}^{m_{1} n_{1} \times m_{1} n_{1}}$ are zero matrices.

If the optimal solution $\hat{\mathbf{x}}$ of the problem (3.2) is known, the HRMS image can be represented as A $\hat{\mathbf{x}}$. In order to find the solution of the problem (3.2), we introduce new auxiliary variables $\mathbf{w}=\mathbf{D x}-P$ and $\mathbf{t}=\mathbf{C x}$ and rewrite this problem as

$$
\min _{\mathbf{x}} \frac{1}{2 N_{u m}}\left\{\|\mathbf{A x}-\mathbf{y}\|_{2}^{2}+\frac{\mu}{2}\|\mathbf{B} \mathbf{x}\|_{2}^{2}+\lambda_{1}\|\mathbf{C x}\|_{1}\right\}+\lambda_{2}\|\nabla \mathbf{w}\|_{1} .
$$

The corresponding augmented Lagrangian function for the problem (3.3) has the form

$$
\begin{aligned}
\mathscr{L}(\mathbf{x}, \mathbf{w}, \mathbf{t})= & \frac{1}{2 N_{u m}}\left\{\|\mathbf{A} \mathbf{x}-\mathbf{y}\|_{2}^{2}+\frac{\mu}{2}\|\mathbf{B} \mathbf{x}\|_{2}^{2}+\lambda_{1}\|\mathbf{C x}\|_{1}\right\}+\lambda_{2}\|\nabla \mathbf{w}\|_{1} \\
& +\frac{\tau}{2}\left\|\mathbf{w}-(\mathbf{D x}-P)+\mathbf{b}_{1}\right\|_{2}^{2}+\frac{\eta}{2}\left\|\mathbf{t}-\mathbf{C x}+\mathbf{b}_{2}\right\|_{2}^{2},
\end{aligned}
$$

where $\mathbf{b}_{1}, \mathbf{b}_{2}$ are the Lagrangian multipliers and $\tau>0, \eta>0$ are the penalty parameters controlling the linear constraints. According to the ADMM method, we shall find a saddle point $\left(\mathbf{x}^{*}, \mathbf{w}^{*}, \mathbf{t}^{*}\right)$ of the function $\mathscr{L}$ in (3.4). The iterative scheme consists of fixing two variables and searching the minimiser with respect to third one. The corresponding ADMM procedure for (3.2) is written as Algorithm 3.1 below.

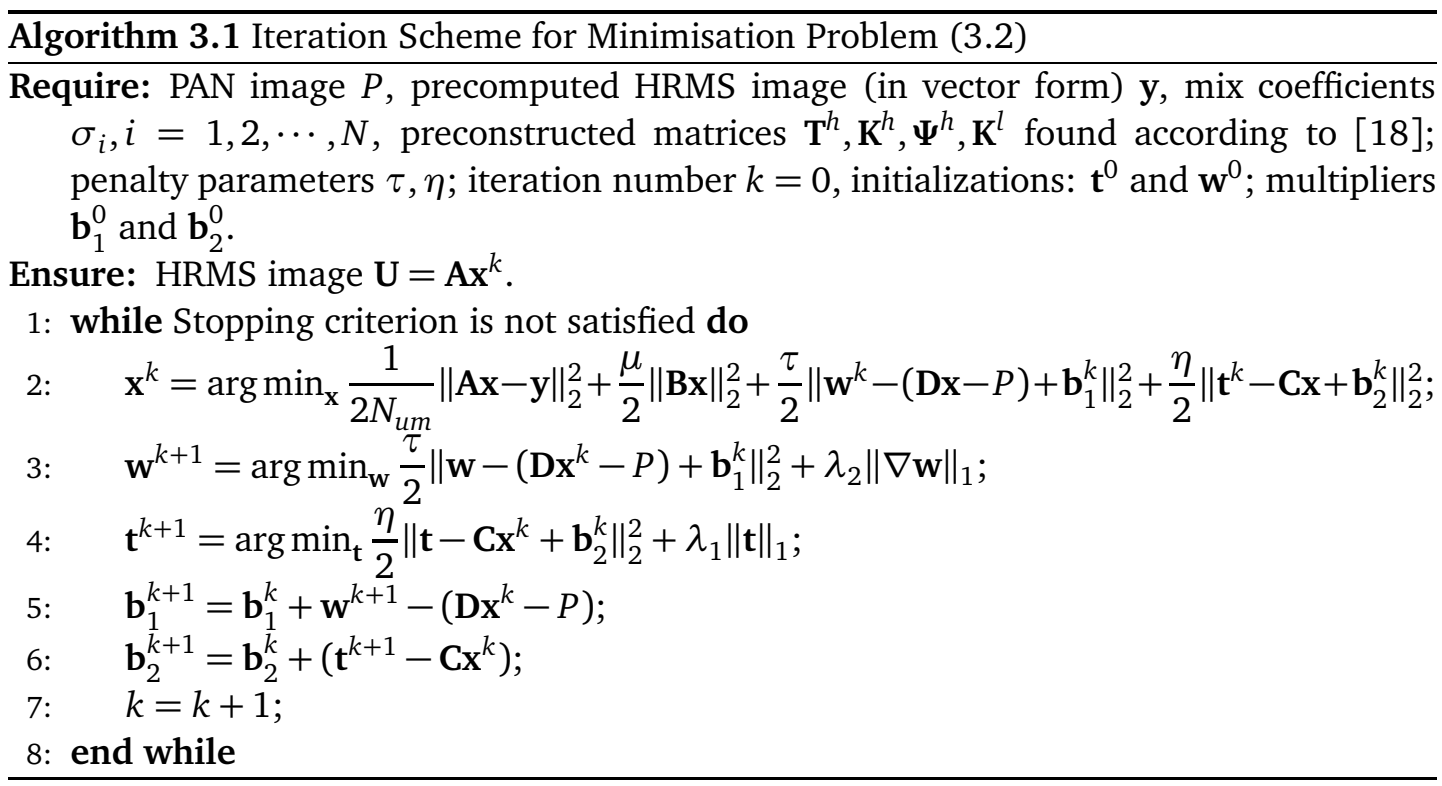


It shows that the minimiser is obtained iteratively and alternatively. We study these subproblems one by one. The minimisation problem with respect to $\mathbf{x}-\mathrm{cf}$. Step 3 in Algorithm 3.1, is a least square problem, equivalent to the following normal equation:

$$
\left(\frac{1}{N_{u m}} \mathbf{A}^{T} \mathbf{A}+\mu \mathbf{B}^{T} \mathbf{B}+\tau \mathbf{D}^{T} \mathbf{D}+\eta \mathbf{C}^{T} \mathbf{C}\right) \mathbf{x}=\frac{1}{N_{u m}} \mathbf{A}^{T} \mathbf{y}+\tau \mathbf{D}^{T}\left(\mathbf{w}^{k}+P+\mathbf{b}_{1}^{k}\right)+\eta \mathbf{C}^{T}\left(\mathbf{t}^{k}+\mathbf{b}_{2}^{k}\right) .
$$

It is clear that finding the inverse matrix for the whole image is not very efficient way to work. Instead, we will handle the pansharpening problem on patches. Thus, the whole image is divided into a number of overlapped patches of a relatively small size and after all patches are worked out, we combine them into a full image. However, we shall be sure that for all patches the inversion operation is feasible.

Step 4 in Algorithm 3.1 is $\mathbf{w}$-subproblem. It can be considered as an $\ell_{2}$-total variation denoising proposed by Rudin, Osher and Fatemi in 1992 [36]. This is why we named our method as Rudin-Osher-Fatemi model based model. Approximate solution of this problem can be found by Chambolle's dual algorithm. We recall that considering an $r \times r$ patch, Chambolle [12] proposed to replace the optimisation of the variable $\mathbf{w}$ by determining a vector field $\mathbf{p}$, which is connected with $\mathbf{w}$ as $\mathbf{w}=\mathbf{D} \mathbf{x}^{k}-P-\lambda_{2} / \tau \operatorname{div} \mathbf{p}$ and is a solution of the minimisation problem

$$
\min _{\mathbf{p}}\left\|\mathbf{D x}^{k}-P-\frac{\lambda_{2}}{\tau} \operatorname{div} \mathbf{p}\right\|_{2}^{2} \text { such that }\left|\mathbf{p}_{i, j}\right| \leq 1 \quad \text { for all } \quad 1 \leq i, j \leq r,
$$

where $\mathbf{p}_{i, j}=\left(\mathbf{p}_{i, j}^{1}, \mathbf{p}_{i, j}^{2}\right)$ is the dual variable at the $i, j$-th pixel location, $\mathbf{p}$ is concatenation of all $\mathbf{p}_{i, j}$, and $\operatorname{div} \mathbf{p} \equiv \mathbf{p}_{i, j}^{1}-\mathbf{p}_{i-1, j}^{1}+\mathbf{p}_{i, j}^{2}-\mathbf{p}_{i, j-1}^{2}$ the discrete divergence of $\mathbf{p}$ with $\mathbf{p}_{0, j}^{1}=\mathbf{p}_{i, 0}^{2}=0$. For simplicity, we set $\alpha=\lambda_{2} / \tau$ and $\mathbf{g}=\mathbf{D x}^{k}-P$. Then the iterative scheme for finding the optimal solution of (3.5) is given by

$$
\mathbf{p}_{i, j}^{l+1}=\frac{\mathbf{p}^{l}+\gamma \alpha \nabla\left(\alpha \operatorname{div} \mathbf{p}^{l}-\mathbf{g}^{l}\right)_{i, j}}{1+\gamma \alpha\left|\nabla\left(\alpha \operatorname{div} \mathbf{p}^{l}-\mathbf{f}^{l}\right)_{i, j}\right|} \quad \text { for all } \quad 1 \leq i, j \leq r,
$$

where $\gamma$ is the step size - cf. [12]. Assuming that $\mathbf{p}^{*}$ is the minimiser of the constrained optimisation problem (3.6), we write the solution of Step 4 in Algorithm 3.1 as

$$
\mathbf{w}^{k+1}=\mathbf{D} \mathbf{x}^{k}-P-\lambda_{2} / \tau \operatorname{div} \mathbf{p}^{*} .
$$

The subproblem for $\mathbf{t}$ - i.e. the Step 5 in Algorithm 3.1 can be solved exactly. More precisely, according to [19], the minimiser of the problem

$$
\arg \min _{s} \frac{1}{2}|z-s|^{2}+\rho|s|, \quad \rho>0
$$

is

$$
s= \begin{cases}z-\rho, & z>\rho \\ 0, & |z| \leq \rho \\ z+\rho, & z<-\rho\end{cases}
$$


It follows that the minimiser $\mathbf{t}_{i}^{k+1}$ of the corresponding subproblem in Step 5 of Algorithm 3.1 can be represented as

$$
\mathbf{t}_{i}^{k+1}= \begin{cases}\left(\mathbf{C} \mathbf{x}^{k}-\mathbf{b}_{2}^{k}\right)_{i}-\frac{\lambda_{1}}{\eta}, & \left(\mathbf{C} \mathbf{x}^{k}-\mathbf{b}_{2}^{k}\right)>\frac{\lambda_{1}}{\eta}, \\ 0, & \left|\left(\mathbf{C} \mathbf{x}^{k}-\mathbf{b}_{2}^{k}\right)\right| \leq \frac{\lambda_{1}}{\eta} \\ \left(\mathbf{C} \mathbf{x}^{k}-\mathbf{b}_{2}^{k}\right)_{i}+\frac{\lambda_{1}}{\eta}, & \left(\mathbf{C} \mathbf{x}^{k}-\mathbf{b}_{2}^{k}\right)<-\frac{\lambda_{1}}{\eta} .\end{cases}
$$

It is clear that Algorithm 3.1 is a particular case of ADMM. It is known [9] that if all subproblems in Steps 3-5 have closed-form solutions, then the method proposed converges. We observe that Step 3 is related to a least squares problem which has a closed-form solution and Step 5 reduces to a soft-thresholding problem [19], which has a closed-form solution. However, it is not always possible to derive an exact solution of the Step 4 problem. On the other hand, one can try to use an alternating splitting with $\mathbf{w}=\nabla \mathbf{D} x-\nabla \mathbf{P}$, but it is difficult to compute the inverse of the matrix related to $(\nabla \mathbf{D})^{T} \nabla \mathbf{D}$. Nevertheless, if the sequence of errors of the successive solutions in Step 3 is absolutely summable, Algorithm 3.1 still converges. The proof of this result can be found in [22, Theorem 8]. Similar considerations are also present in [9].

It is easily seen that the solution of the proposed model (3.1) is related to the precomputed HRMS image Ũ. To improve the accuracy, we can update the scheme in Algorithm 3.1 as is noted in Algorithm 3.2. The updating strategy of Algorithm 3.2 has been used by several image applications, including natural image super-resolution [18] and remote sensing image pansharpening [41]. Algorithm 3.2 can be considered as the acquisition of details from an LRMS image for the use in the HRMS image. The downsample step in Algorithm 3.2 is defined as follows: $\tilde{\mathbf{u}}^{l}$ is first filtered by a Gaussian filter matched with the modulation transfer function (MTF) and then downscaled by a "nearest" interpolation to $\mathbf{v}^{l}$ having the same size as the LRMS image $\mathbf{u}$.

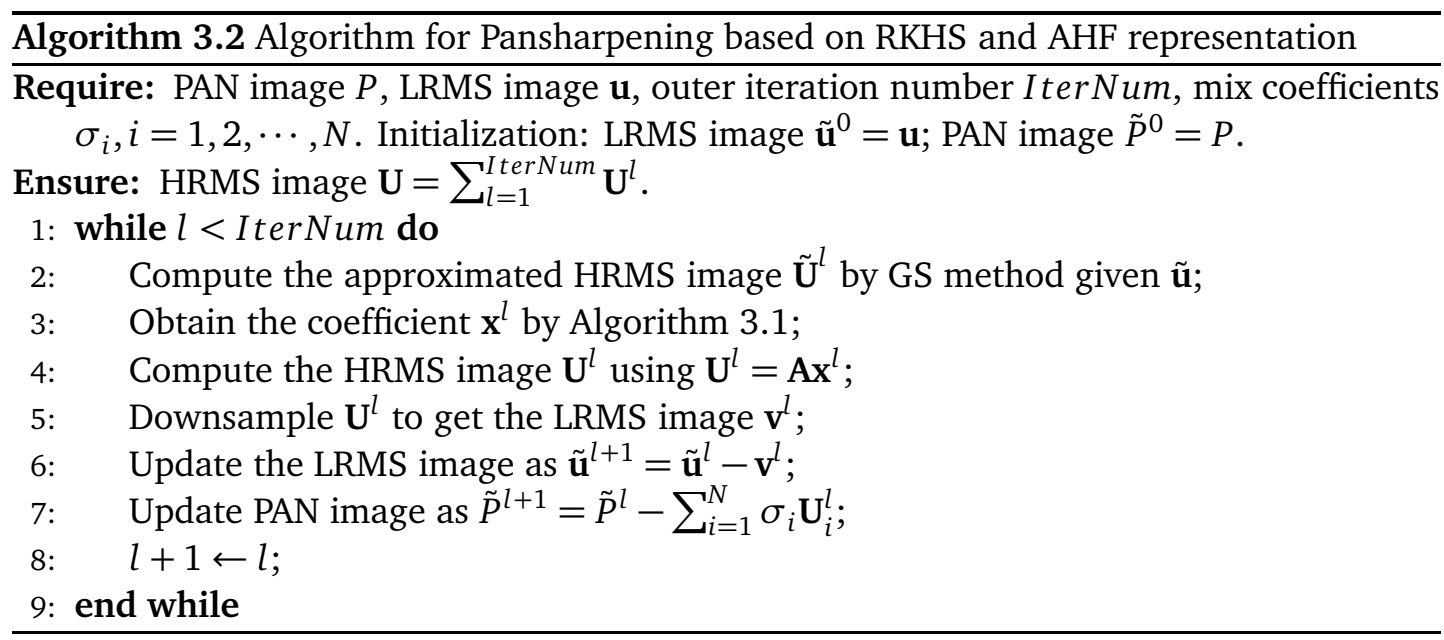




\section{Experiments}

In this section, we provide numerical results demonstrating the performance of the method. Various pansharpening methods are tested on the data from Pléiades and IKONOS satellites. For both satellite data, the MS images have four bands - viz. blue, green, red and near-infrared ones. Pléiades dataset can be downloaded from public web "Open Remote Sensing" - cf. https://openremotesensing.net/knowledgebase/a-criticalcomparison-among-pansharpening-algorithms. For Pléiades dataset details see [5] and references therein. The IKONOS dataset can be can be found at the Global Land Cover Facility web page http://glcf.umd.edu/data. This web page also has MS and PAN data from other satellites. The images in both datasets are very large. Therefore, for experiments only small portions of the original images are used. For Pléiades dataset, we crop the $64 \times 64 \times 4$ and $256 \times 256$ upper left portion of the low resolution multi-spectral bands and PAN image. Based on this dataset, we also manually set simulated test data e.g. the high-resolution panchromatic image is obtained by the average of green and red bands of the HRMS image. The LRMS bands with four times lower spatial resolution than of the panchromatic one, are simulated according to MTF filtering and decimation. Let us call this simulated dataset PléiadesSimu. The test data from this dataset is obtained in the same way as the previous one. For the data acquired by the IKONOS satellites, we choose the mountainous and vegetated area of the Sichuan region in China. The experimental data are collected similarly to Pléiades dataset but the cropped LRMS bands and PAN image are, respectively, of the size $32 \times 32 \times 4$ and $128 \times 128$.

The experiments are implemented in MATLAB(R2017a) environment on MacBook pro PC with 8Gb RAM and Intel(R) Core(TM) CPU i5: @3.1 GHz. The parameters in the algorithm are chosen as $\mu=1.2 \times 10^{5}, \lambda_{1}=2 \mu, \lambda_{2}=\mu, \tau=\mu / 100, \eta=\mu / 1000$. The mix coefficients $\sigma_{i}, i=1,2,3,4$ are estimated by the linear regression of the MS bands and the spatially degraded PAN image [3]. The dimension $m$ of the polynomial space is 6 , the number of orientations $M=45$ and 3-iterated Laplacian is used. The outer iterations IterNum and the Chambolle's denoising step are both equal to 5 . To reduce computation cost and the storage size, we apply our method to image patches with $6 \times 6$ of 5 pixels patches overlap.

Our method is compared with ten popular pansharpening methods - viz. EXP [1] (MS image interpolation, using a polynomial kernel with 23 coefficients), BDSD [26] (BandDependent Spatial-Detail with local parameter estimation), GS [30] (Gram Schmidt), PRACS [15] (Partial Replacement Adaptive Component Substitution), HPF [34] (High-Pass Filtering with $5 \times 5$ box filter for fusion), Indusion [29] (Decimated Wavelet Transform using an additive injection model), ATWT [42] (Additive a Trous Wavelet Transform with unitary injection model), AWLP [33] (Additive Wavelet Luminance Proportional), MTF-GLP-CBD (Generalized Laplacian Pyramid (GLP) [1] with MTF-matched filter [2] and regression based injection model [5]), ICIP-RKHS [16]. Note that [16] was the first work, where

RKHS was used for pansharpening. The reader can also find an extension of ICIP-RKHS from [17]. The codes of the algorithms mentioned are freely available at the web page https://openremotesensing .net/knowledgebase/a-critical-comparison- 
among-pansharpening-algorithms.

Figs. 1(b)-1(1) show the pansharpening for Pléiades dataset. For visual convenience, we only show the blue, green and red bands of the HRMS image obtained. It is clear that the fused HRMS image obtained by our method is competitive. It outperforms other methods in terms of spatial and spectral fidelity with respect to the ground truth image. CS-based method experiences significant loses spectral information while preserving spatial details. The MRA-based method AWLP does not demonstrate good results in preserving spatial information. On the other hand, the method we propose does preserve spatial details and recover high-quality spectral information very well.

Fig. 2 demonstrates the fused images for IKONOS-China. Note a spectral distortion in the images obtained by GS and ATWT methods. On the other hand, the HRMS images obtained by ICIP-RKHS and our method have a better spatial and spectral quality - e.g. the boundary of the river and the surface of the mountain are much clearer and more vivid.

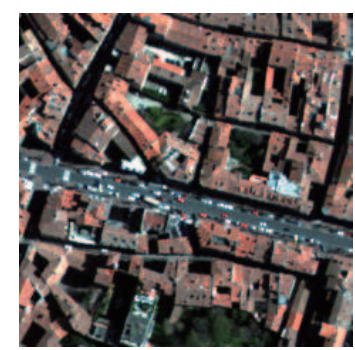

(a) GT

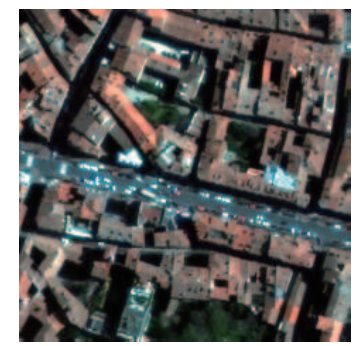

(e) PRACS

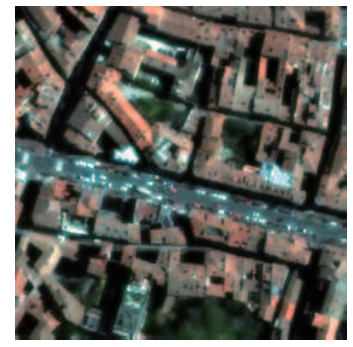

(i) AWLP

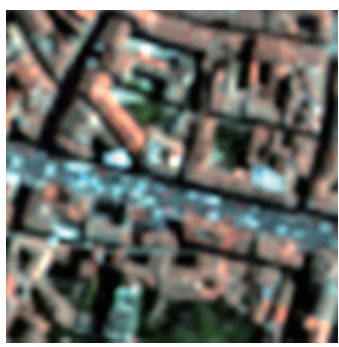

(b) EXP

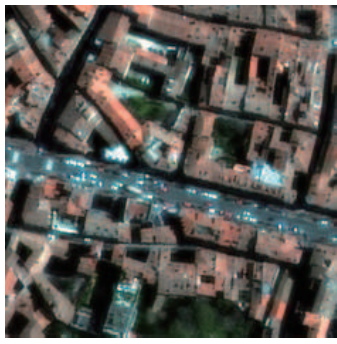

(f) $\mathrm{HPF}$

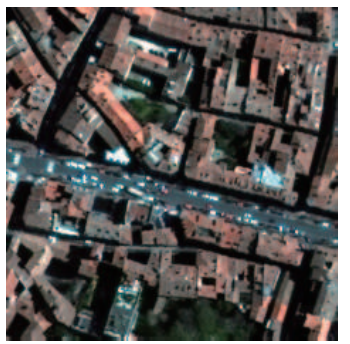

(j) MTF-GLP-CBD

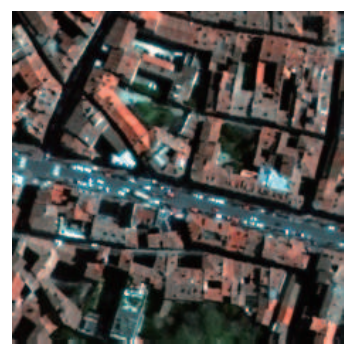

(c) BDSD

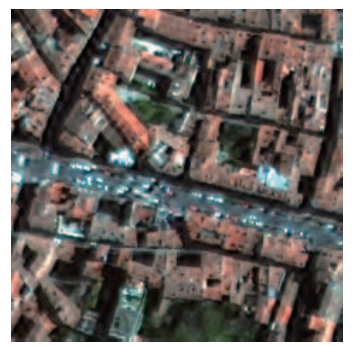

(g) Indusion

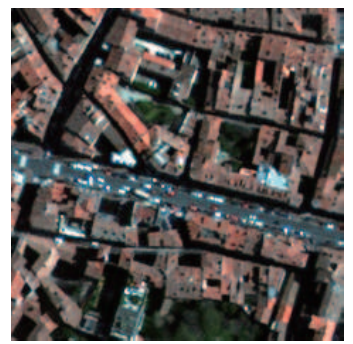

(k) ICIP-RKHS

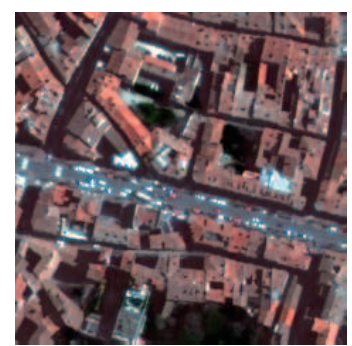

(d) GS

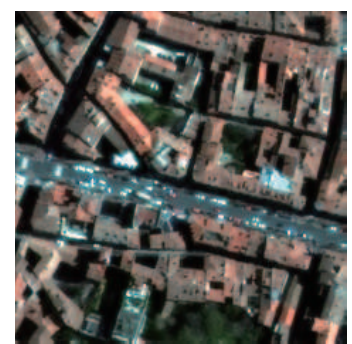

(h) ATWT

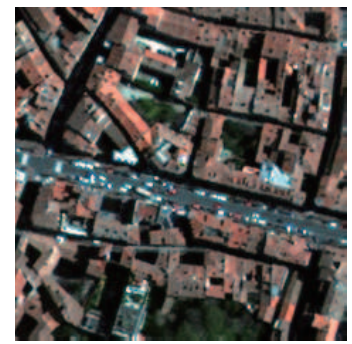

(1) Ours

Figure 1: Works of various methods on Pléiades dataset. GT denotes ground truth image. 


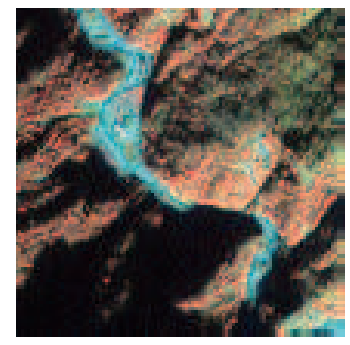

(a) GT

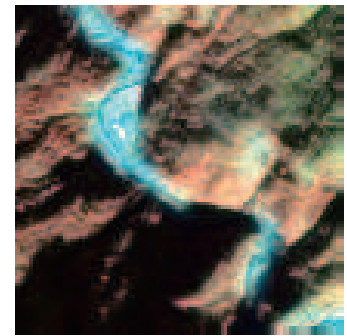

(e) PRACS

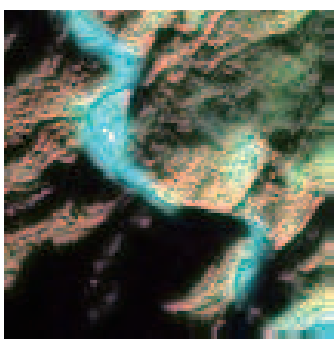

(i) AWLP

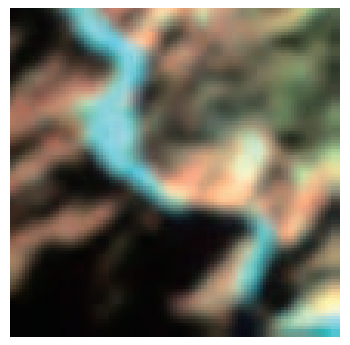

(b) EXP

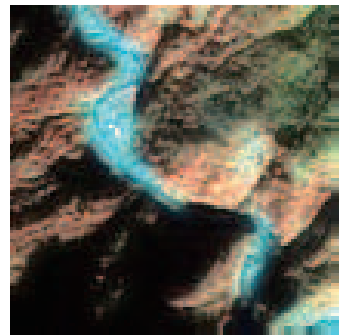

(f) $\mathrm{HPF}$

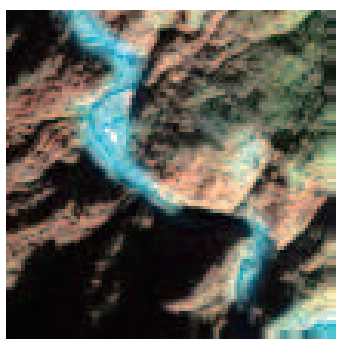

(j) MTF-GLP-CBD

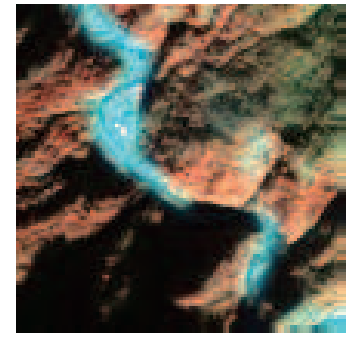

(c) BDSD

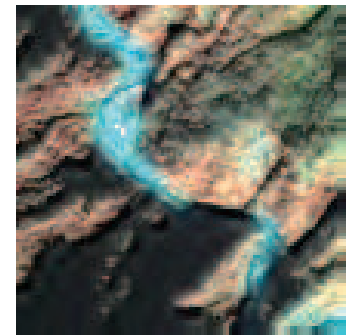

(g) Indusion

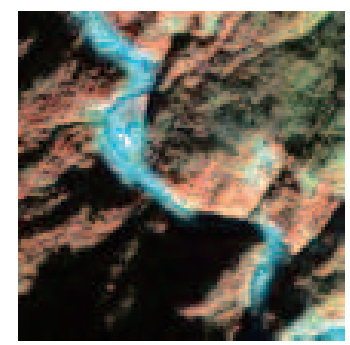

(k) ICIP-RKHS

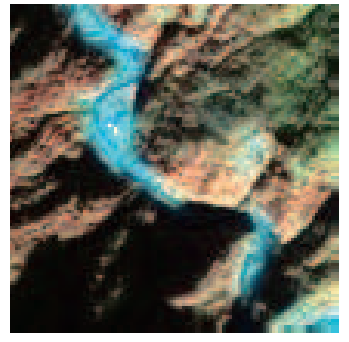

(d) GS

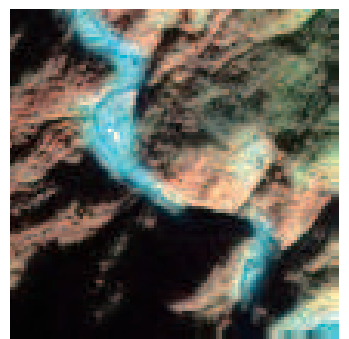

(h) ATWT

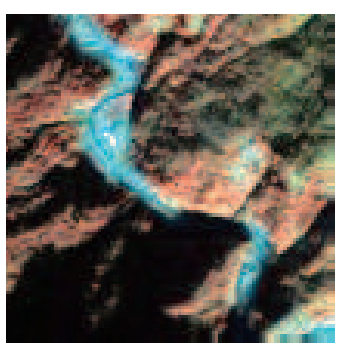

(1) Ours

Figure 2: GT denotes ground truth image. Comparisons of different methods on IKONOS-China dataset.

For quantitative assessments of the fusion we use the Q4 vector index [4], Q index [45], Spectral Angle Mapper (SAM) [46], Erreur Relative Globale Adimensionnelle de Synthèse (ERGAS) [44], and Spatial Correlation Coefficient (SCC). The results are demonstrated in Tables 1-3, where bold highlights the best performance. Overall, the quality of fused images obtained by the method proposed are higher than for other methods. Moreover, they show that the RKHS and AHF based representations are practically useful for pansharpening and that the ROF based model outperforms the least square model.

Let us also discuss the role of various parameters in Algorithm 3.2, using Pléiades dataset as a base for their testing. For simplicity, in experiments we only take the topleft part of size $128 \times 128 \times 4$. In order to independently evaluate the parameter sensitivity, we fix all parameters except the one we want to study. To study the impact of the number of outer iterations on the performance of the method, we empirically set it to 1, 5, 25 and 30 and evaluate the corresponding changes in different measurements. The results 
Table 1: PléiadesSimu dataset. Bold highlights the best performance.

\begin{tabular}{||c|c|c|c|c|c||}
\hline & Q4 & Q & SAM & ERGAS & SCC \\
\hline EXP [1] & 0.7878 & 0.7629 & 4.6685 & 5.7915 & 0.6500 \\
\hline BDSD [26] & 0.9765 & 0.9734 & 3.4606 & 2.3104 & 0.9413 \\
\hline GS [30] & 0.8975 & 0.9040 & 4.7511 & 3.9996 & 0.9325 \\
\hline PRACS [15] & 0.9511 & 0.9449 & 3.8740 & 3.0323 & 0.9283 \\
\hline HPF [34] & 0.9462 & 0.9397 & 3.6215 & 3.1337 & 0.9234 \\
\hline Indusion [29] & 0.8924 & 0.8894 & 4.5325 & 4.3842 & 0.8658 \\
\hline ATWT [42] & 0.9631 & 0.9571 & 3.2633 & 2.6712 & 0.9366 \\
\hline AWLP [33] & 0.9617 & 0.9533 & 3.2526 & 2.7201 & 0.9294 \\
\hline MTF-GLP-CBD [5] & 0.9773 & 0.9730 & 3.1081 & 2.2222 & 0.9422 \\
\hline ICIP-RKHS [16] & 0.9756 & 0.9725 & 3.0782 & 2.2628 & 0.9424 \\
\hline Our Method & $\mathbf{0 . 9 8 1 2}$ & $\mathbf{0 . 9 7 8 2}$ & $\mathbf{2 . 7 5 6 5}$ & $\mathbf{2 . 0 1 4 0}$ & $\mathbf{0 . 9 5 1 7}$ \\
\hline
\end{tabular}

Table 2: Pléiades dataset. The best results are highlighted by black bold.

\begin{tabular}{||c|c|c|c|c|c||}
\hline & Q4 & Q & SAM & ERGAS & SCC \\
\hline EXP [1] & 0.7878 & 0.7629 & 4.6685 & 5.7915 & 0.6500 \\
\hline BDSD [26] & 0.9733 & 0.9703 & 3.6455 & 2.4611 & 0.9358 \\
\hline GS [30] & 0.8943 & 0.9004 & 4.7790 & 4.0834 & 0.9264 \\
\hline PRACS [15] & 0.9419 & 0.9354 & 3.9436 & 3.2840 & 0.9210 \\
\hline HPF [34] & 0.9413 & 0.9348 & 3.7075 & 3.2726 & 0.9185 \\
\hline Indusion [29] & 0.8897 & 0.8866 & 4.5589 & 4.4465 & 0.8612 \\
\hline ATWT [42] & 0.9585 & 0.9525 & 3.3764 & 2.8409 & 0.9305 \\
\hline AWLP [33] & 0.9571 & 0.9487 & 3.3590 & 2.8760 & 0.9236 \\
\hline MTF-GLP-CBD [5] & 0.9735 & 0.9694 & 3.3009 & 2.4051 & 0.9367 \\
\hline ICIP-RKHS [16] & 0.9710 & 0.9704 & 3.2752 & 2.3928 & $\mathbf{0 . 9 4 4 6}$ \\
\hline Our Method & $\mathbf{0 . 9 7 7 1}$ & $\mathbf{0 . 9 7 4 2}$ & $\mathbf{2 . 9 7 5 8}$ & $\mathbf{2 . 2 2 0 6}$ & 0.9417 \\
\hline
\end{tabular}

Table 3: IKONOS-China dataset. The best results are highlighted by black bold.

\begin{tabular}{||c|c|c|c|c|c||}
\hline & Q4 & Q & SAM & ERGAS & SCC \\
\hline EXP [1] & 0.8006 & 0.7916 & 5.2953 & 4.4520 & 0.6793 \\
\hline BDSD [26] & 0.9237 & 0.9149 & 3.3210 & 2.8000 & 0.9215 \\
\hline GS [30] & 0.8934 & 0.8890 & 4.3686 & 3.0929 & 0.9186 \\
\hline PRACS [15] & 0.9196 & 0.9085 & 3.6495 & 2.8542 & 0.9104 \\
\hline HPF [34] & 0.9141 & 0.9018 & 3.7200 & 2.9162 & 0.9066 \\
\hline Indusion [29] & 0.8374 & 0.8473 & 4.6643 & 3.8194 & 0.8705 \\
\hline ATWT [42] & 0.9244 & 0.9114 & 3.4225 & 2.7483 & 0.9182 \\
\hline AWLP [33] & 0.9247 & 0.9107 & 3.3312 & 2.7629 & 0.9170 \\
\hline MTF-GLP-CBD [5] & 0.9251 & 0.9129 & 3.1978 & 2.7015 & 0.9214 \\
\hline ICIP-RKHS [16] & 0.9206 & 0.9071 & 3.3591 & 2.7870 & 0.9106 \\
\hline Our Method & $\mathbf{0 . 9 2 8 2}$ & $\mathbf{0 . 9 1 8 7}$ & $\mathbf{3 . 1 6 2 9}$ & $\mathbf{2 . 6 7 7 4}$ & $\mathbf{0 . 9 2 1 8}$ \\
\hline
\end{tabular}




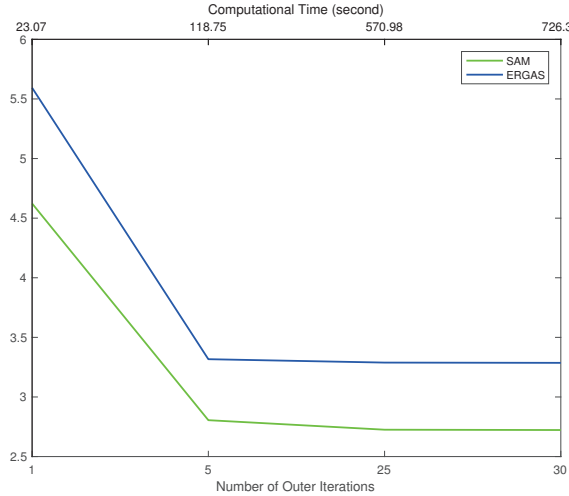

(a)

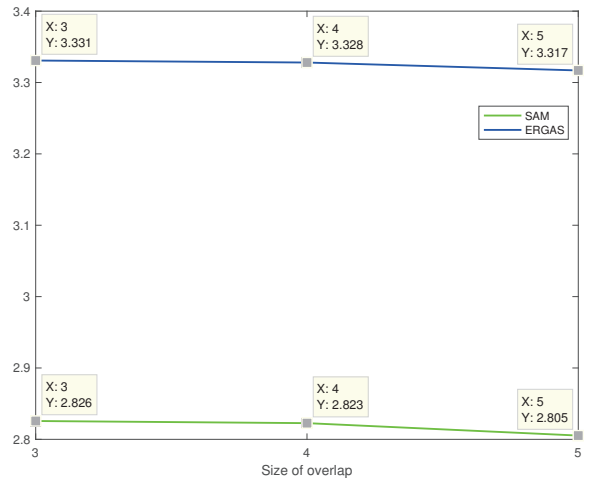

(c)

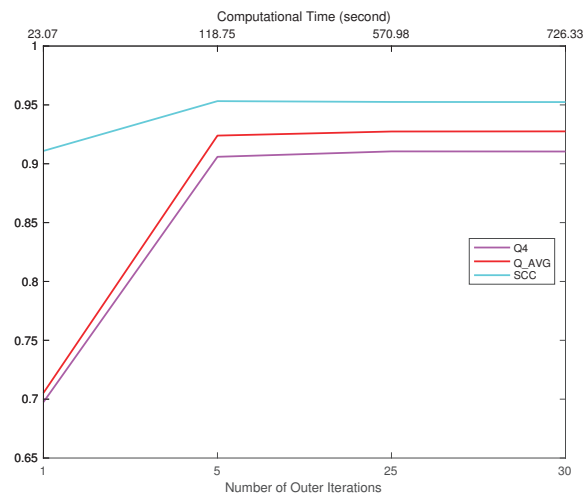

(b)

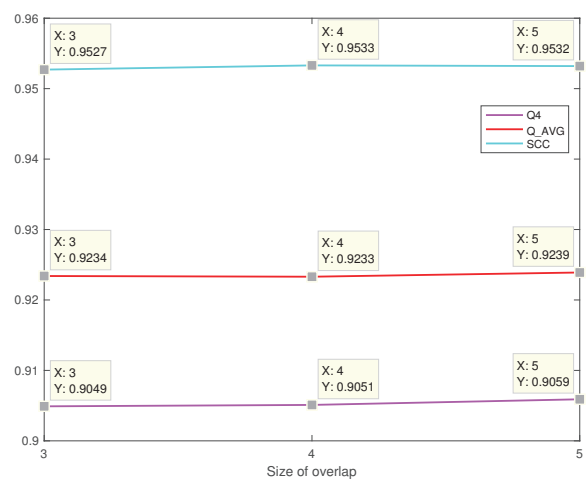

(d)

Figure 3: Pansharpening for various outer iterations and overlapping size in Algorithm 3.2.

are shown in Figs. 3(a) and 3(b). The worst results are for outer iteration number 1, but they got slightly better as iteration proceeds. However, in all experiments we set the number of outer iterations to 5 to balance computational cost and performance. Figs. 3(c) and 3 (d) present the results with the patch overlap of different size. We observe that the best performance is achieved for the overlap equals 5 .

\section{Conclusion}

We studied the pansharpening problem based on the Rudin-Osher-Fatemi model by using RKHS and AHF representation and proposed an efficient algorithm under the framework of the classic ADMM method. To demonstrate the effectiveness of the method, we carried out numerous experiments on Pléiades and IKONOS datasets and compared the results with those obtained by ten known pansharpening methods. It turns out that the method we propose is able to construct an HRMS image and preserves the spectral and spatial information. 


\section{Acknowledgments}

This work is supported in part by the NSFC (Grants Nos. 11701079, 61871185, 61702083), by the Jilin Provincial STDP (Grants Nos. 20170520053JH,20170520050JH), by the Jilin Provincial EDSRP (JJKH20190293KJ), by the Fundamental Research Funds

for the Central Universities ( ZYGX2016KYQD142) and by the China Postdoctoral Science Foundation (2016M601360). Shanghai Chenguang Program (17CG25).

\section{References}

[1] B. Aiazzi, L. Alparone, S. Baronti and A. Garzelli, Context-driven fusion of high spatial and spectral resolution images based on oversampled multiresolution analysis, IEEE Trans. Geosci. Remote 40, 2300-2312 (2002).

[2] B. Aiazzi, L. Alparone, S. Baronti, A. Garzelli and M. Selva, Mtf-tailored multiscale fusion of high-resolution ms and pan imagery, Photogramm. Eng. Rem. S. 72, 591-596 (2006).

[3] B. Aiazzi, S. Baronti and M. Selva, Improving component substitution pansharpening through multivariate regression of MS +Pan data, IEEE Trans. Geosci. Remote 45, 3230-3239 (2007).

[4] L. Alparone, S. Baronti, A. Garzelli and F. Nencini, A global quality measurement of pansharpened multispectral imagery, IEEE Geosci. Remote S. 1, 313-317 (2004).

[5] L. Alparone, L. Wald, J. Chanussot, C. Thomas, P. Gamba and L.M. Bruce, Comparison of pansharpening algorithms: Outcome of the 2006 GRS-S data-fusion contest, IEEE Trans. Geosci. Remote. Sens. 45, 3012-3021 (2007).

[6] I. Amro, J. Mateos, M. Vega, R. Molina and A.K. Katsaggelos, A survey of classical methods and new trends in pansharpening of multispectral images, EURASIP J. Adv. Sig. Pr. 79, (2011).

[7] V.A. Aydin and H. Foroosh, Volumetric super-resolution of multispectral data, Arxiv 1705.05745 (2017).

[8] C. Ballester, V. Caselles, L. Igual, J. Verdera and B. Rougé, A variational model for $P+X S$ image fusion, Int. J. Comput. Vision 69, 43-58 (2006).

[9] J.M. Bioucas-Dias and M.A.T. Figueiredo, Multiplicative noise removal using variable splitting and constrained optimization, IEEE Trans. Image Process. 19, 1720-1730 (2010).

[10] P.J. Burt and E.H. Adelson, The Laplacian pyramid as a compact image code, Readings in Computer Vision 31, 532-540 (1983).

[11] W.J. Carper, The use of intensity-hue-saturation transformations for merging spot panchromatic and multispectral image data, Photogramm. Eng. Rem. S. 56, 459-467 (1990).

[12] A. Chambolle, An algorithm for total variation minimization and applications, J. Math. Imaging Vision 20, 89-97 (2004).

[13] A. Chambolle and T. Pock, A first-order primal-dual algorithm for convex problems with applications to imaging, J. Math. Imaging Vision 40, 120-145 (2011).

[14] C. Chen, Y. Li, W. Liu and J. Huang, Image fusion with local spectral consistency and dynamic gradient sparsity, Computer Vision and Pattern Recognition (CPVR), pp. 2760-2765, IEEE (2014).

[15] J. Choi, K. Yu and Y. Kim, A new adaptive component-substitution-based satellite image fusion by using partial replacement, IEEE Trans. Geosci. Remote. Sens. 49, 295-309 (2010).

[16] L.J. Deng, V. Gemine, W. Guo, D.M. Mauro and C. Jocelyn, A variational pansharpening approach based on reproducible kernel hilbert space and heaviside function, International Conference on Image Processing (ICIP), pp. 535-539, IEEE (2017). 
[17] L.J. Deng, V. Gemine, W. Guo, D.M. Mauro and C. Jocelyn, A variational pansharpening approach based on reproducible kernel Hilbert space and Heaviside function, IEEE Trans. Image Process. 27, 4330-4344 (2018).

[18] L.J. Deng, W. Guo and T. Z. Huang, Single-image super-resolution via an iterative reproducing kernel Hilbert space method, IEEE Trans. Circuits Syst, 26, 2001-2014 (2016).

[19] D. L. Donoho, De-noising by soft-thresholding, IEEE Trans. Inform. Theory 41, 613-627 (1995).

[20] J. Duchon, Splines minimizing rotation-invariant semi-norms in Sobolev spaces, Springer (1977).

[21] J. Duran, A. Buades, B. Coll, C. Sbert and G. Blanchet, A survey of pansharpening methods with a new band-decoupled variational model, ISPRS J. Photogramm 125, 78-105 (2017).

[22] J. Eckstein and D. P. Bertsekas, On the Douglas-Rachford splitting method and the proximal point algorithm for maximal monotone operators, Math. Program. 55, 293-318 (1992).

[23] F. Fang, F. Li, C. Shen and G. Zhang, A variational approach for pan-sharpening, IEEE Trans. Image Process. 22, 2822-2834 (2013).

[24] D. Fasbender, J. Radoux and P. Bogaert, Bayesian data fusion for adaptable image pansharpening, IEEE Trans. Geosci. Remote. Sens. 46, 1847-1857 (2008).

[25] D. Gabay and B. Mercier, A dual algorithm for the solution of nonlinear variational problems via finite element approximation, Comput. Math. Appl, 2, 17-40 (1976).

[26] A. Garzelli, F. Nencini and L. Capobianco, Optimal mmse pan sharpening of very high resolution multispectral images, IEEE Trans. Geosci. Remote. Sens. 46, 228-236 (2007).

[27] R. Glowinski and A. Marroco, Sur l'approximation, par éléments finis d'ordre un, et la résolution, par pénalisation-dualité d'une classe de problèmes de Dirichlet non linéaires, Revue française d'automatique, informatique, recherche opérationnelle, analyse numérique 9, 41-76 (1975).

[28] Y. Jiang, X. Ding, D. Zeng, Y. Huang and J. Paisley, Pan-sharpening with a hyper-Laplacian penalty, International Conference on Computer Vision (ICCV), pp. 540-548, IEEE (2015).

[29] M.M. Khan, J. Chanussot, L. Condat and A. Montanvert, Indusion: Fusion of multispectral and panchromatic images using the induction scaling technique, IEEE Geosci. Remote S. 5, 98-102 (2008).

[30] C.A. Laben and B. V. Brower, Process for enhancing the spatial resolution of multispectral imagery using pansharpening, US patent 6011875 (2000).

[31] J. Liu, T. Z. Huang, I. W. Selesnick, X. G. Lv and P. Y. Chen, Image restoration using total variation with overlapping group sparsity, Inform. Sci, 295, 232-246 (2015).

[32] M. Möller, T. Wittman, A. L. Bertozzi and M. Burger, A variational approach for sharpening high dimensional images, SIAM J. Imaging Sci. 5, 150-178 (2012).

[33] X. Otazu, M. Gonzalez-Audicana, O. Fors and J. Nunez, Introduction of sensor spectral response into image fusion methods. Application to wavelet-based methods, IEEE Trans. Geosci. Remote. Sens. 43, 2376-2385 (2005).

[34] C. Psjr, S.C. Sides and J.A. Anderson, Comparison of three different methods to merge multiresolution and multispectral data: Landsat tm and spot panchromatic, Photogramm. Eng. Rem. S. 57, 265-303 (1991).

[35] R. Restaino, G. Vivone, M.D. Mura and J. Chanussot, Fusion of multispectral and panchromatic images based on morphological operators, IEEE Trans. Image Process. 25, 2882-2895 (2016).

[36] L.I. Rudin, S. Osher and E. Fatemi, Nonlinear total variation based noise removal algorithms, Phys. D 60, 259-268 (1992).

[37] R.A. Schowengerdt, Reconstruction of multispatial, multispectral image data using spatial frequency content, Photogramm. Eng. Rem. S. 46, 1325-1334 (1980).

[38] M.J. Shensa, The discrete wavelet transform: wedding the a trous and mallat algorithms, IEEE Trans. Signal Process. 40, 2464-2482 (1992). 
[39] C. Thomas, T. Ranchin, L. Wald and J. Chanussot, Synthesis of multispectral images to high spatial resolution: A critical review of fusion methods based on remote sensing physics, IEEE Trans. Geosci. Remote. Sens. 46, 1301-1312 (2008).

[40] T.M. Tu, P.S. Huang, C.L. Hung and C.P. Chang, A fast intensity-hue-saturation fusion technique with spectral adjustment for ikonos imagery, IEEE Geosci. Remote S. 1, 309-312, (2004).

[41] M.R. Vicinanza, R. Restaino, G. Vivone, M.D. Mura, G. Licciardi and J. Chanussot, A method for improving the consistency property of pansharpening algorithms, IEEE Geoscience and Remote Sensing Symposium, pp. 2534-2537, IEEE (2014).

[42] G. Vivone, R. Restaino, M.D. Mura, G. Licciardi and J. Chanussot, Contrast and error-based fusion schemes for multispectral image pansharpening, IEEE Geosci. Remote S. 11, 930-934 (2014).

[43] G. Wahba, Spline models for observational data (CBMS-NSF Regional Conference series in applied mathematics), Watson Research Center, 59 (1990).

[44] L. Wald, Data fusion. definitions and architectures-fusion of images of different spatial resolutions, Les Presses de l' École des Mines(2002).

[45] Z. Wang and A.C. Bovik, A universal image quality index, IEEE Signal Proc. Let. 9, 81-84 (2002).

[46] R. Yuhas, A.F.H. Goetz and J.W. Boardman, Descrimination among semi-arid landscape endmembers using the spectral angle mapper (SAM) algorithm, in Proceeding Summaries 3rd Annual JPL Airborne Geoscience Workshop. 1, pp. 147-149, NASA (1992).

[47] Z.Y. Zhang, T.Z. Huang, L.J. Deng, J. Huang, X.L. Zhao and C.C. Zheng, A framelet-based iterative pan-sharpening approach, Remote Sens. 10, 622 (2018). 